\title{
Nonlinear and negative resistance in loose electrical contacts dark bridge
}

\author{
Hiromichi Kubota, Shunsuke Sasaki, Hiroyuki Ishida, Tasuku Takagi* \\ Graduate School of Health and Environment Sciences,Tohoku Bunka Gakuen University, Sendai, Japan \\ Email address: \\ tasuku@sirius.ocn.ne.jp(T. Takagi)
}

\section{To cite this article:}

Hiromichi Kubota, Shunsuke Sasaki, Hiroyuki Ishida, Tasuku Takagi. Nonlinear and Negative Resistance in Loose Electrical Contacts Dark Bridge. American Journal of Physics and Applications. Vol. 1, No. 1, 2013, pp. 1-4. doi: 10.11648/j.ajpa.20130101.11

\begin{abstract}
The dark bridge in loose electrical contacts was investigated, which grew up when the contact gap was increased a little with micron by micron. The dark bridge was watched by microscopic camera along with measurement of voltage-current (V-I) relationship. At very small gap (less than $19 \mu \mathrm{m}$ for iron (Fe) contacts but different from materials), the V-I characteristics were linear, which is normal, but more than the $20 \sim 30 \mu \mathrm{m}$, the non-linear relationship appeared and finally the negative resistance was observed. Experimental growth of dark bridge was shown in case of Pd. The nonlinear and negative resistance phenomena were tested for Fe. The resistance was analyzed and showed that it goes to negative one after crossing zero from positive region.
\end{abstract}

Keywords: Electrical Contacts, Dark Bridge, Nonlinear, Exponential, Negative Resistance

\section{Introduction}

Electrical contacts are indispensable for every electrical system of which degradation due to contact failure is common. The degradation can be simulated by very accurate electrodes separation system using cantilever[1]. Separation gap was less than $1 \mu \mathrm{m}$ step by step with a sufficient time so that the contact spot temperature reaches the thermal equilibrium condition[2], and we got the gap from several to more than ten micrometers but still current continues to flow through bridge (named dark bridge[1]). In the practical situation, the thermal stress can deform the contact spot[3] and the non-luminous bridge (dark bridge) may be produced to maintain the current flow. If the contact system carries the digital signal along with power current, this situation of contacts may degrade the reliability of communication system[4].

This paper describes the phenomena observed on the dark bridge, which we have never experienced so far about the characteristic examination of the dark bridge. We, at the beginning, wanted to know the resistance of the dark bridge, because the bridge is so small that must have a big resistance which may disturb the flow of current. But the result was quite different and not simple. There happened to appear not only nonlinear voltage vs. current (V-I) characteristics but also negative resistance. The typical materials, Pd and Fe were tested. Pd was examined how the dark bridge grows, and for Fe, the resistance was examined from V-I characteristics.

\section{Observed Images and Size of Dark Bridge}

The images of dark bridge were taken for $\mathrm{Pd}$ and $\mathrm{Fe}$ contacts. Both images are shown in Fig.1 (a) and (b) respectively. The size of the bridge is the order of several micrometers $(\mu \mathrm{m})$ for $\mathrm{Pd}$ and that for $\mathrm{Fe}$ is far bigger (about ten times) than that of Pd. The size difference may stem from the material properties because the current was almost the same with each other.

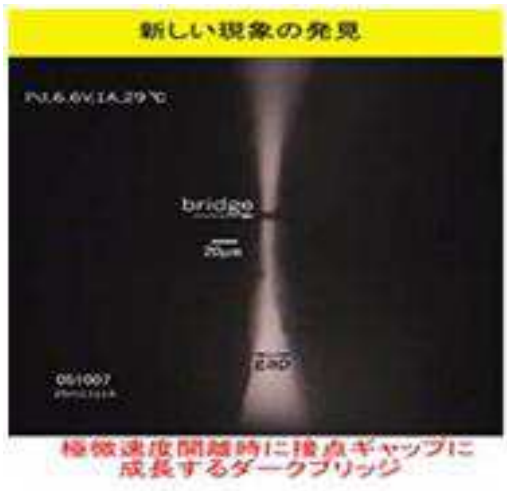

Figure 1. (a) Pd dark bridge (electrode diameter is $1 \mathrm{~mm} \varphi$ ) 


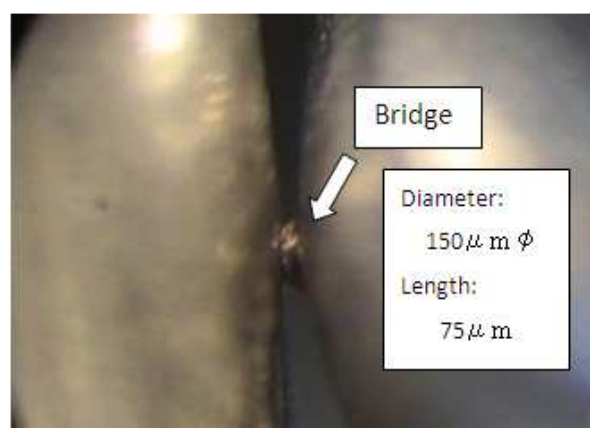

Figure 1. (b) Fe dark bridge

The size of dark bridge can be estimated from enlarged image, and shown in Fig. 2 for Pd. The relationship between bridge length and diameter was approximated by the exponential function as shown in Fig.2.

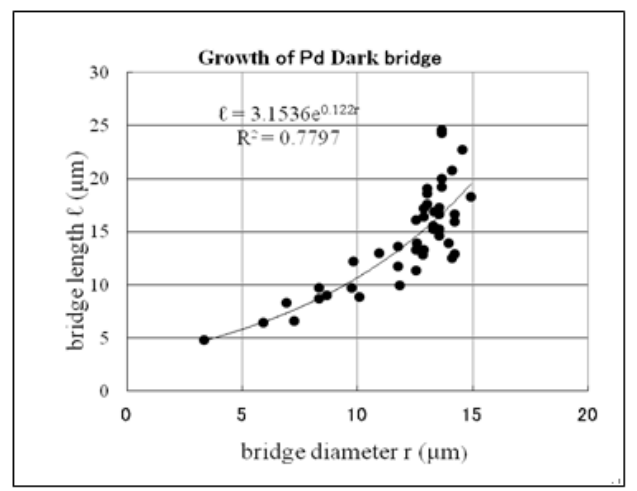

Figure 2. Size of dark bridge for Pd (length vs. diameter)

\section{V-I Characteristics of Fe Dark Bridge}

\subsection{Measurement Circuit System and Image Acquisition}

Hereafter, we shall show in case of Fe. Figure. 3 shows the circuit system for measurement of V-I characteristics of Fe dark bridge. The contact current I is supplied by the voltage source through current meter and resistor (wire resistance loop: $5 \Omega$ ) to the contacts of which voltage $\mathrm{V}$ is measured by the voltmeter. The microscopic camera was used to observe the images of dark bridge. The images were stored in the computer. The separation gap of electrodes is recorded so that we can know how much separation gap grew up.

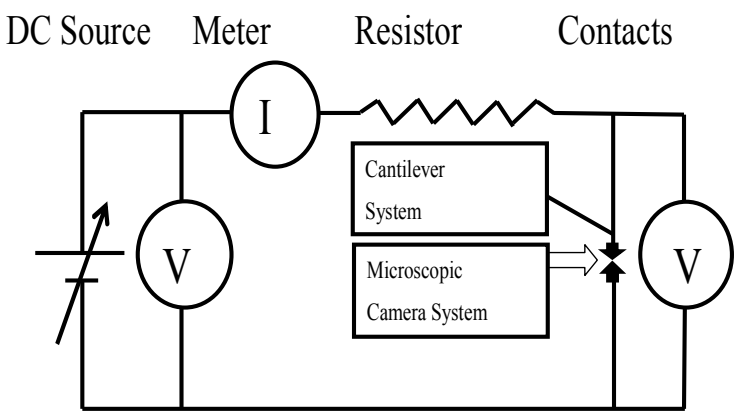

Figure 3. Circuit Diagram for Measurement of Dark Bridge
Observing the contacts during separation, the micro-gap discharges intermittently occurred. The discharge phenomena of this type is known as ESD (Electro-Static Discharge) which is known as a very hazardous to the digital communication networks[4]. The recording of contact voltage was stored in the PC.

\subsection{V-I Characteristics}

Figure 4 shows the obtained V-I characteristics. Clearly we can see that Fig. 4 has two types of characteristics; one group is linear relationship and another one is non-linear one where the curves (encircled 1 and 2) are those of the first data and the second one respectively. The authors wondered when looked at first curve (encircled 1) if the data were erroneous. Then, again tried and we got the curve 2. The results were the same shape. Thus we are convinced of the integrity of the data.

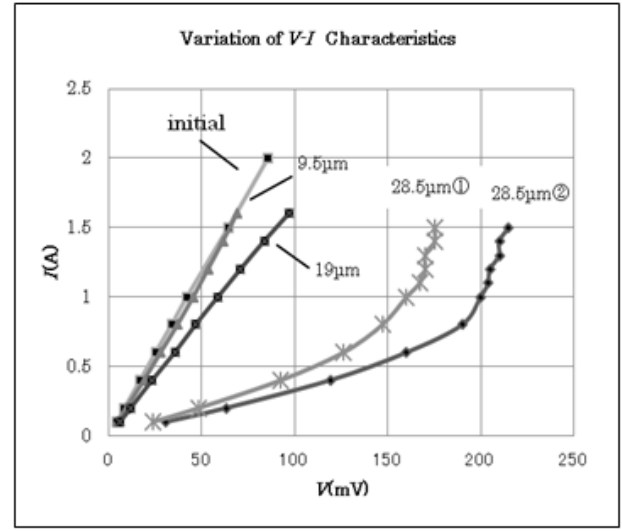

Figure 4. V-I characteristics of Fe dark bridge

The linear group is easy to understand. The 'initial' in Fig.4 is the beginning of our experiments; the linear relationship was obtained. The line of $9.5 \mu \mathrm{m}$ was almost the same with the initial one. But with more $9.5 \mu \mathrm{m}$ separation to $19 \mu \mathrm{m}$, the slope became a little gentle, which means the conductance became small (resistance increased). The linearity of V-I relationship for contact resistance is normal and common. However, in case where the electrode separated to $28.5 \mu \mathrm{m}$, the $\mathrm{V}-\mathrm{I}$ relationship changed drastically as shown in Fig.4.

\subsection{Outlook of V-I Characteristics}

As mentioned above, V-I characteristics shown in Fig.4 have divided into two types: Linear and Nonlinear.

The Linear type ('initial' to ' $19 \mu \mathrm{m}$ ') has shown the normal electrical contacts phenomena. That is the current constriction resistance property[5]. That is to say, contact resistance increases when contact force decreases. Fig.4 shows that the contact resistance increases (conductance decreases) with respect to contact force which decreases due to the electrodes separation. But quantitative discussion on this matter should be necessary in future.

The Nonlinear type (' $28.5 \mu \mathrm{m}$ ') is not simple. We have to carefully scrutinize the phenomena as clear as possible. 
Here, we can say the following things to be made clear: The nonlinear curves can be seen that they are consisted of the three regions. We analyze the first one (curve encircled 1) of nonlinear curves shown in Fig.5, which can be partitioned by regions of linear (A-B), upward curve (B-C), and of unstable (C-D), which should be all analyzed quantitatively.

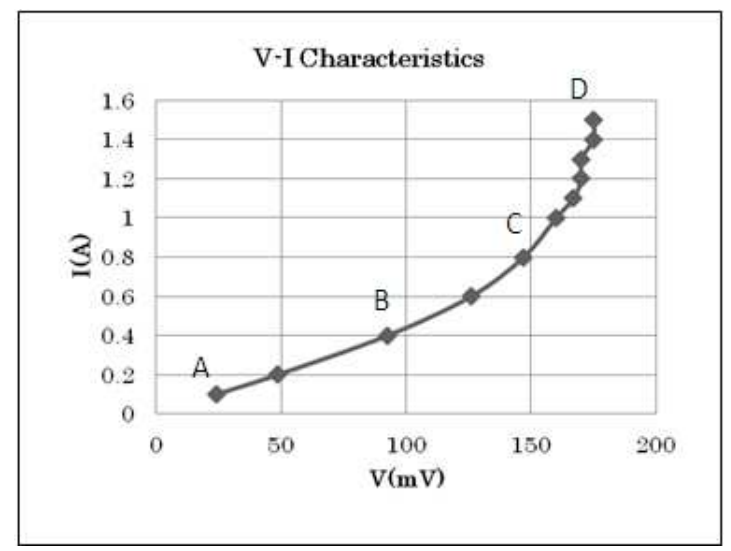

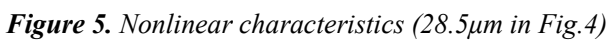

\section{Analyses of Linear and Nonlinear}

\subsection{Characteristics of Linear Group}

Here, the three straight lines in Fig. 4 are the subject. The slope of straight characteristics can be seen as a normal metal V-I relationship. The unit of the coefficient of slope is Siemens (S) that means conductance. Since the inverse unit of $S$ is $\Omega$ (resistance), from the three characteristics of the 'initial' to ' $19 \mu \mathrm{m}$ ' in Fig.4, we can see that the resistance of ' $19 \mu \mathrm{m}$ ' increased from those of 'initial' and ' $9.5 \mu \mathrm{m}$ ' (both have almost the same resistance). The increase of resistance can be interpreted from R.Holm's theory: This is due to the decrease of contact area so called a-spot[5].

The resistance of 'initial' and ' $19 \mu \mathrm{m}$ ' are (in $\mathrm{m} \Omega$ );

initial:45

$19 \mu \mathrm{m}: 60$

This means the diameter of a-spot area (contact point was assumed to be circle) decreased by the square root of $45 / 60$.

\subsection{Characteristics of Nonlinear Part - Analysis of Partitioned Region}

Here we pick up the first nonlinear curve (encircled 1) in Fig.4 for convenience, which is shown in Fig.5, where we partitioned the curve into three regions as shown like A-B, $\mathrm{B}-\mathrm{C}$, and C-D. This partition was made by the result of try and cut analyses. We shall show what rules exist in each partitioned region. The results are as follows:

Region A-B showed the linear, B-C was exponential, and C-D was unstable, where we obtained the resistance variation with respect to voltage that revealed the negative resistance after it passes through the zero resistance. Those evidences will be mentioned hereafter.
The result can be summarized that both regions of A-B and B-C are clearly we can say from computer analyses that both regions are those of almost perfect linear and exponential characteristics each other. But the region C-D is quite complex as shown later.

Region A-B: The characteristic of A-B region looks linear. This is true. The result is shown in Fig.6. This region is linear but the resistance is larger than those of the Linear Group shown in the above 4.1. As shown in Fig.6, the computer approximation shows the conductance of this region is $0.0034(\mathrm{mS})$ that means $0.294(\Omega)$ which is much larger than those of the Linear Group shown in 4.1.

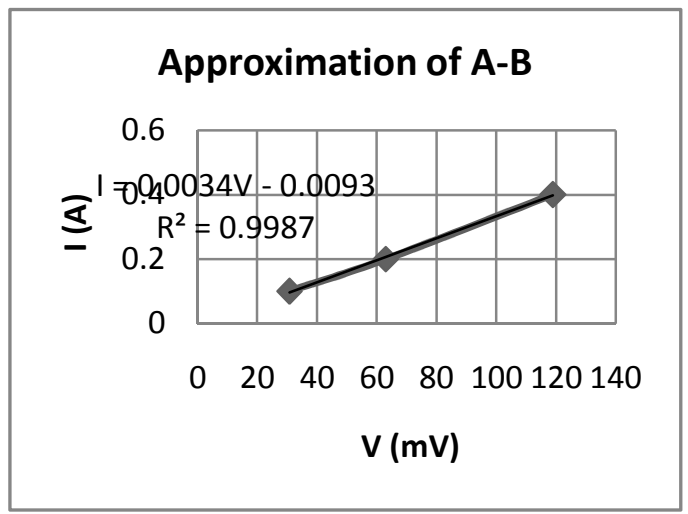

Figure 6. Linear approximation of region $A-B$

0.0093 in the formula in Fig.6 is error, because when $\mathrm{V}=0$, I should be zero. The accuracy of the approximation is good, because the squared correlation index $\mathrm{R}^{2}$ is very close to $1 . \mathrm{R}^{2}=1$ means the phenomenon can perfectly obeys the physical law (Ohm's Law).

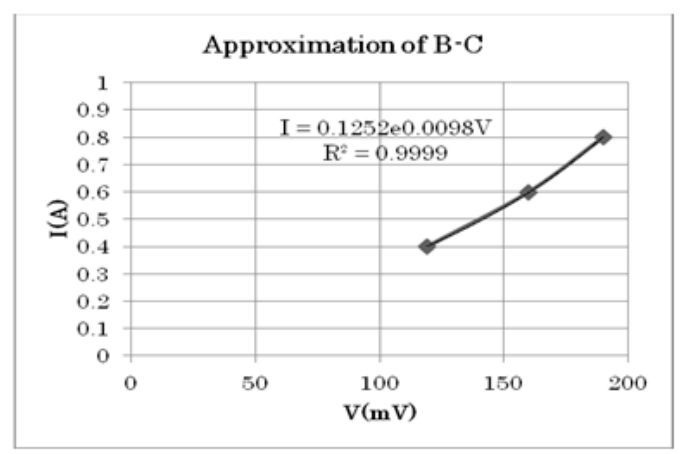

Figure 7. Exponential approximation of region $B-C$

Region B-C: This region was approximated by theexponential function. As shown in Fig.7, the approximation was also so accurate that the correlation factor $\mathrm{R}^{2}$ was almost 1 as shown. The reason why the dark bridge can be exponential is the future subject, but we know that our natural phenomena always obey the law of the exponential function. This case can be considered from such a viewpoint.

Region C-D: According to the experiment, we were not able to get the stable V-I characteristics, because the reading of meter could not stop and unstable. The plotted 
marks in this region in Fig. 4 were medium values that were observed. In order to get more information on this phenomenon, we tried another experiments. Fig. 8 is the one of the results.

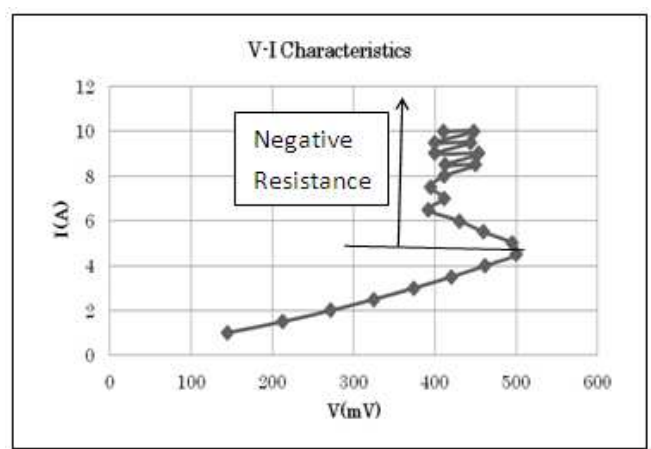

Figure 8. V-I characteristics with negative resistance

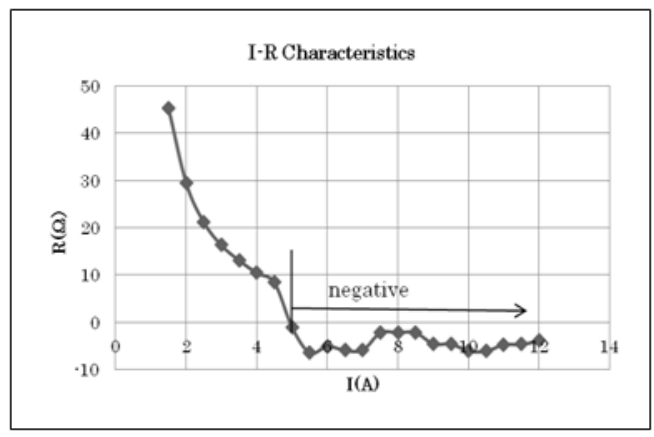

Figure 9. Negative resistance in iron dark bridge

Fig. 8 shows the abnormal curve with negative resistance. The smooth upward curve looks natural, but after $\mathrm{V}=500 \mathrm{mV}$, the curve abruptly turned to decrease to $400 \mathrm{mV}$. After that, V-I characteristics cannot be stable. We assume those unstable phenomena should be stems from the appearance of the negative resistance. The voltage is decreasing but the current increasing, which means this region should be of negative resistance. If we calculate the derivatives of this curve shown in Fig.8, we get Fig.9, which clearly shows the negative resistance region. This is the very rear phenomenon, but the same test has tried for
$\mathrm{Pd}$ and $\mathrm{Cu}$ and we have had the same phenomena.

We know the negative resistance is active, which can create an oscillatory phenomenon, such as a semiconductor tunnel diode or PNPN device. It is very interesting to have a very simple device of metal contact dark bridge just mentioned has the same characteristics.

\section{Conclusion}

We saw the dark bridge characteristics in case of Fe. How dark bridge grows was confirmed. The nonlinear and negative resistance were discovered and analyzed. We are now going to test the other metals including Pd of which dark bridge growing property was confirmed as shown in Fig. 2 but the unstable region has not been scrutinized yet. Essential matters in the future are relationships between material constants and the nonlinearity.

\section{References}

[1] Hiroyuki Ishida, Masanari Taniguchi, Tasuku Takagi, "Precise Measurement of Dark Bridge between Micro-gap Electrical Contacts in a State of Thermal Equilibrium Condition", Proc. of the IEEE IMTC 2005, Ottawa Canada May 2005.

[2] Hiroyuki Ishida, Masanari Taniguchi, Hideaki Sone, Hiroshi Inoue, Tasuku Takagi, "Relationship between Length and Diameter of Contact Bridge Formed under Thermal Equliburium Condition", IEICE Transactions on Electronics, Vol. E88-C ,No.8, 2005

[3] Hiromichi Kubota, Masanari Taniguchi, Shosuke Suzuki, Tasuku Takagi, "Contact Failure and Thermal Deformation Analysis of Printed Circuit Board Connector Due to Thermal Stress by Using Holography,", Proceedings of the ICEC2008 Saint-Malo-France, 2008

[4] TAKAGI Tasuku, "Noise properties in breaking and loose contacts and their effect on quality degradation of digital signal", Journal of Zhenjiang University SCIENCE A 2007 $8(3): 357-360$

[5] R.Holm, Electric Contacts: theory and applications,p.11 16, Springer-Verlag, Berlin 2000 